\title{
Proteasome Inhibitors In Cancer Therapy: A Novel Approach To A Ubiquitous Problem
}

\author{
KRISTIN R LANDIS-PIWOWAR
}

\section{LEARNING OBJECTIVES:}

1. Describe the basic structure and function of the proteasome.

2. Identify the proteasomal subunit within the catalytic core that is crucial to cell survival.

3. List proteasome protein targets.

4. Evaluate the effect on cellular homeostasis following proteasome inhibition.

5. Detail the status of various proteasome inhibitors in pre-clinical and clinical trials.

ABSTRACT: The cellular proteasome is an important molecular target in cancer therapy and drug resistance research. Proteasome inhibitors are effective agents against multiple myeloma and mantle cell lymphoma and display great potential as treatment for a variety of other malignancies. The proteasome is a large multicatalytic, proteinase complex located in the cytosol and the nucleus of eukaryotic cells. The ubiquitin proteasome system is responsible for the degradation of most intracellular proteins and therefore plays an essential regulatory role in critical cellular processes including cell cycle progression, proliferation, differentiation, angiogenesis, and apoptosis. Cancer cells are particularly sensitive to proteasome inhibitors, indicating the utility for inhibition of the ubiquitinproteasome pathway as an approach for cancer therapy.

INDEX TERMS: proteasome, ubiquitin, apoptosis, multiple myeloma, clinical trials

Clin Lab Sci 2012;25(1):38

Kristin R. Landis-Piwowar, PhD,MT(ASCP), Oakland University, School of Health Sciences, Biomedical Diagnostic and Therapeutic Sciences, Rochester, MI

Address for Correspondence: Kristin Landis-Piwowar, Ph.D. MT (ASCP), School of Health Sciences, 321 HHS, Oakland University, 2200 N. Squirrel Rd, Rochester, MI
48309,248-370-4039, landispi@oakland.edu

\section{INTRODUCTION}

The cancer-preventive and anticancer effects of dietary polyphenols and flavonoids such as those in green tea are supported by results from epidemiological, cell culture, and animal studies. Several of these plant polyphenols act as proteasome inhibitors and may sensitize tumor cells to traditional chemo- and radiation therapies by interfering in pathways. While chemotherapy is an established means for cancer management, the accompanying systemic toxicity and potential for regression or complete ineffectiveness remain as real obstacles for cancer patients. To surmount these obstacles, targeting specific molecules, as opposed to the mass destruction of all mitotically active cells, has found favor as a unique treatment option in certain cancers. In fact, the proteasome has been implicated as an important molecular target in cancer and drug resistance research. Bortezomib (codeveloped by Millenium Pharmaceuticals Inc., Cambridge, MA, USA and Johnson \& Johnson Pharmaceutical Research and Development, L.L.C., Raritan, NJ, USA), the first and currently only proteasome inhibitor approved by the US Food and Drug Administration, is used most commonly as a combination agent for the treatment of both multiple myeloma and mantle cell lymphoma. ${ }^{1}$ However, numerous clinical trials are underway to study the effects of bortezomib in a variety of other malignancies as well.

Bortezomib has been shown to improve clinical outcomes in certain cancers, ${ }^{2,3}$ yet cancer cell resistance and the predictable toxicity associated with bortezomib treatment, raises the need to identify novel, synthetic or naturally occurring proteasome inhibitors. This article describes the basic physiology and biological function of the proteasome, the known proteasome protein targets and the effect of their accumulation upon proteasome 


\section{FOCUS: ADVANCES IN CLINICAL CANCER RESEARCH}

inhibition, and details the status of various proteasome inhibitors in pre-clinical and clinical trials.

\section{The Ubiquitin Proteasome Pathway}

Proteolysis in eukaryotic cells occurs predominantly within the ubiquitin (Ub)-proteasome pathway. The proper function of this pathway is a critical physiological event that maintains the intracellular concentration of a variety of proteins. Disruption of appropriate proteasome activity is disastrous to cellular homeostasis. ${ }^{4}$ The Ub-proteasome pathway involves conjugation of activated ubiquitin molecules to a protein substrate, and the ultimate degradation of that Ub-tagged protein by the proteasome. Ubiquitin is a 76-amino acid protein that is covalently ligated to a target protein by a multi-enzymatic system. ${ }^{5} \mathrm{Ub}$ must be activated, conjugated, and ligated by the sequential actions of E1, E2, and E3 enzymes. E3 enzymes possess a complex mechanism of substrate specificity. The result of this pathway is a protein that is "tagged" with Ub molecules (Figure 1). ${ }^{6}$
Ubiquitinated proteins are recognized and discriminately degraded by the eukaryotic $26 \mathrm{~S}$ proteasome, a massive $(2000 \mathrm{kD})$ multi-catalytic, multi-subunit protease complex located in the nucleus and cytosol of nearly all cells. ${ }^{7}$ Typically, proteins containing polyubiquitin chains (four or more Ub molecules) on lysine 48 are directed to the proteasome fordegradation. ${ }^{8}$ Upon recognition of the $\mathrm{Ub}$ tagged protein by the proteasome, proteolysis of the substrate commences and the $\mathrm{Ub}$ molecules are released and recycled.

The proteolytic core of the $26 \mathrm{~S}$ proteasome, the $20 \mathrm{~S}$ proteasome, is composed of four heptameric, stacked rings $(\alpha 7, \beta 7, \beta 7, \alpha 7)$ that form a barrel-like structure and possess multiple peptidase activities (Figure. 1). ${ }^{9}$ Within the $\alpha$-rings, the $\alpha$-subunits ensure that only unfolded polypeptides are allowed exposure to the central $\beta$-rings. Proteolysis results from substrate contact with the $\beta$-subunits. The $\beta 5$ subunit possess chymotrypsin-like activity and cleaves after amino acids

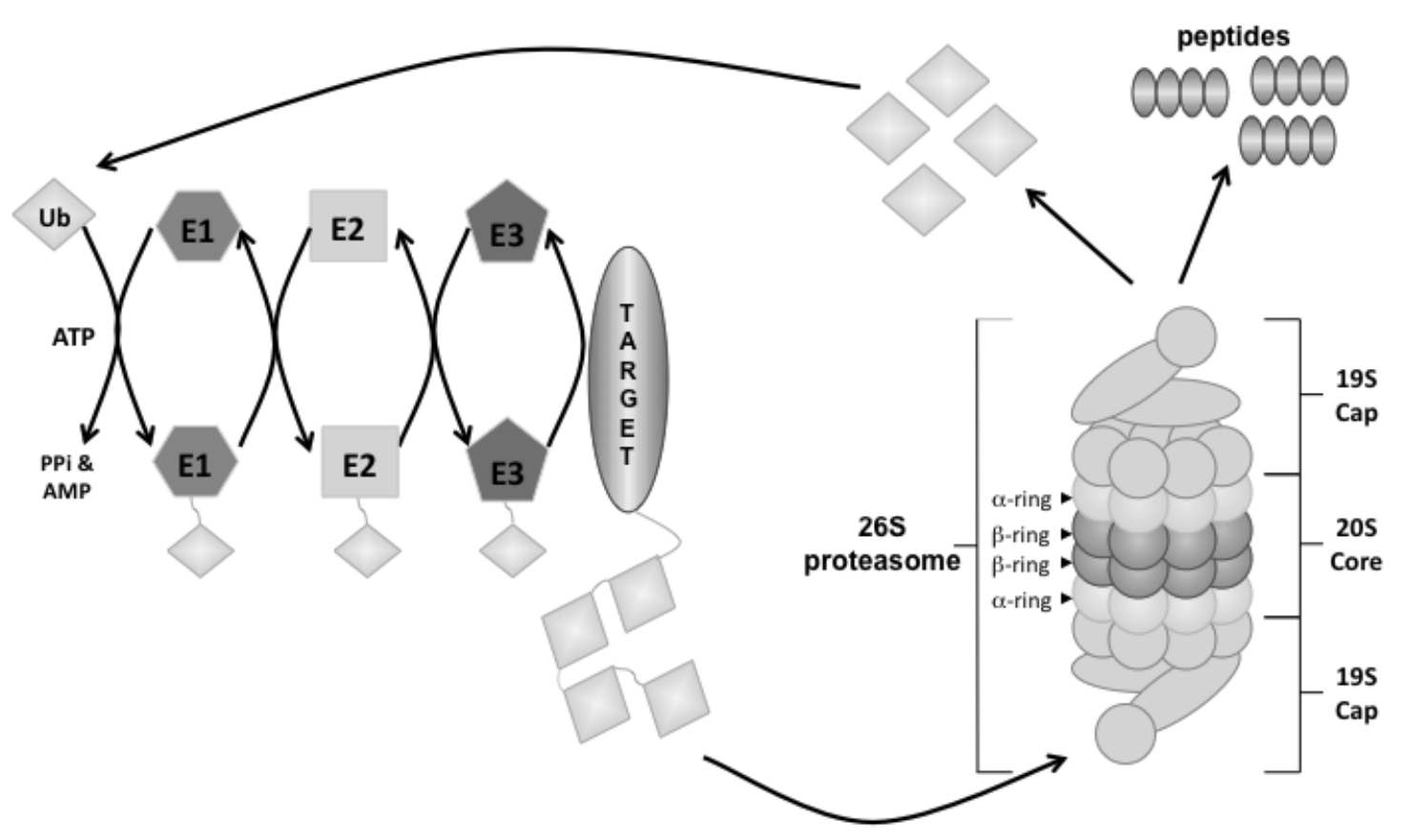

Figure 1. The ubiquitin-proteasome pathway. Ubiquitin (Ub) is ligated to target proteins by a multi-enzymatic system consisting of Ubactivating (E1), -conjugating (E2), and -ligating (E3) enzymes. Ubiquitinated proteins are escorted to the 26S proteasome, through the $19 \mathrm{~S}$ cap and to the catalytic $20 \mathrm{~S}$ core for degradation into individual amino acids or oligopeptides. The Ub is released and recycled to begin the process anew. Adapted from, Landis-Piwowar KR et al. ${ }^{1}$ 


\section{FOCUS: ADVANCES IN CLINICAL CANCER RESEARCH}

with hydrophobic side chains. The $\beta 1$ subunit confers peptidylglutamyl peptide hydrolyzing-like and cleaves after amino acids with acidic side chains. The $\beta 2$ subunit has trypsin-like activity and cleaves after amino acids with basic side chains. ${ }^{9}$ As the polypeptide is sequentially degraded, the by-products formed consist of short polypeptides that range in length from as little as 4 amino acids to 25 amino acids with the average being 7-9 amino acids. ${ }^{10}$ This very carefully controlled system that determines the demise of proteins via ubiquitination, followed by proteasomal degradation is crucial for cellular homeostasis.

\section{Proteasome Substrates and Cellular Consequences of Inhibition}

Because the proteasome is fundamental to maintaining the appropriate balance of cellular proteins it is rather intuitive that disruption of proteasome function would be catastrophic to the fate of a cell. The ubiquitinproteasome pathway is responsible for the degradation of proteins linked to the cell cycle, ${ }^{11-14}$ to apoptosis ${ }^{15}$ and to the accumulation of abnormal proteins that result from cellular stress such as oxidative damage. ${ }^{16}$ Inappropriate proteasome activity can alter cellular homeostasis by progressive degradation of regulatory proteins or stabilization of inappropriately produced proteins. In either case, the proteasome may be implicated in the pathogenesis of various disease states including cancer. ${ }^{17}$ Proteasome inhibition is an established means for the treatment of multiple myeloma, mantle cell lymphoma, and non-Hodgkin lymphoma. ${ }^{3}$ Furthermore, the collection of empirical data for the use of proteasome inhibition as a treatment for various solid tumors is underway. ${ }^{18}$

To fully understand the molecular consequences of proteasome inhibition, a discussion of the specific classes of proteins that are ubiquitinated and degraded by the Ub-proteasome pathway must be included. Proteasome inhibition leads to an almost immediate decrease in the rate of cellular proteolysis. Numerous proteins involved in the formation and the survival of cancer cells have been identified as proteasome substrates and include cell cycle-promoting (cyclins A, $\mathrm{B}, \mathrm{D}$ and $\mathrm{E})^{11-14}$ and inhibiting ( 27$)$ proteins, ${ }^{19}$ the "guardian of the genome" protein (tumor suppressor protein $\mathrm{p} 53),{ }^{20}$ pro-apoptotic protein $\mathrm{Bax},{ }^{15}$ and the inhibitor of NFkB, IkB-a. ${ }^{21}$ Some of these target proteins are discussed below.
The Ub-proteasome pathway closely regulates degradation of proteins (called cyclins) involved in progression through interphase of the cell cycle. For example, both cyclin D and cyclin $\mathrm{E}$ are degraded by ubiquitin-mediated proteolysis and their destruction allows for cell passage out of $G_{1}$ and through to $S$ phase. ${ }^{12,13}$ Likewise, degradation of cyclin $\mathrm{A}$ allows passage from $S$ into $G_{2}$. Finally, for the cell to mitose, cyclin B must be rapidly degraded by the Ubproteasome pathway. ${ }^{11}$ Accumulation of these proteins following proteasome inhibition leads to a halt in cell cycle progression and ultimately to cell death.

Cell survival is critically dependent upon the proper ratio of pro-apoptotic (proteins that incite cell death) to anti-apoptotic proteins (proteins that block cell death). The pro-apoptotic protein $\mathrm{Bax}$ is involved in mitochondrial cytochrome $\mathrm{c}$ release that induces the mechanisms of cell death. ${ }^{22}$ In normal cells, Bax is maintained at relatively low concentration and degradation of Bax by the Ub-proteasome pathway ensures that cells survive to maintain tissue homeostasis. However, in pathological conditions such as cancer, degradation of Bax may be increased ${ }^{15,23-26}$ to promote survival and proliferative ability of the cell ${ }^{15}$ under conditions that would normally be unfavorable. Proteasome inhibitors have been shown to increase the concentration of Bax in cancer cells $s^{27}$ and since highly proliferating cells (cancer cells) are more sensitive to proteasome inhibition than non-proliferating cells, ${ }^{28-32}$ this accumulation of Bax leads to apoptosis induction. ${ }^{1,33}$

The tumor suppressor protein p53 functions in cell cycle regulation and prevention of genome mutation to the extent that it has taken the moniker, "guardian of the genome." p53 is vitally important to blocking cell cycle progression at the $G_{1} / S$ restriction point in the presence of DNA damage and in doing so, promotes apoptosis. Much like Bax, p53 is maintained at a constant, but low level in normal cells under normal conditions and is also targeted by the Ub-proteasome pathway for degradation. ${ }^{34}$ The mechanisms of cancer cell transformation are known to involve altered cell cycle regulation and ineffective DNA repair beyond the control of p53. However, an accumulation of p53 is observed in the presence of proteasome inhibitors 35,36 and leads to the induction of apoptosis. ${ }^{36}$ 


\section{FOCUS: ADVANCES IN CLINICAL CANCER RESEARCH}

Finally, NF-kB (nuclear factor kappa-light-chainenhancer of activated $\mathrm{B}$ cells) is a transcription factor that regulates genes involved in cell survival and proliferation and is aberrantly expressed in numerous conditions, but most importantly for this discussion, in cancer cells. Importantly, the Ub-proteasome pathway does not regulate NF-kB directly, but does so indirectly by degradation of an inhibitory molecule called $\mathrm{IkBa}$ (inhibitor of NF-kB). Very simply, IkBa complexes with NF-kB and in doing so, inhibits the translocation of NF-kB into the nucleus where it could promote transcription of cell survival genes. $\mathrm{IkBa}$ is a target of the Ub-proteasome pathway and is accumulated upon proteasome inhibition. The accumulation of $\mathrm{IkBa}$ sequesters NF-kB in the cytoplasm and in doing so, contributes to apoptosis in cancer cells. ${ }^{37-39}$

\section{Proteasome inhibitors}

Proteasome inhibitors can be categorized as reversible and irreversible and their inhibition is predominately and most commonly accomplished by interaction with the b-subunits. More precisely, inhibition of the $\mathrm{N}$ terminal threonine of the proteasomal $\beta 5$-subunit (chymotrypsin-like activity) has been specifically implicated in conferring apoptosis induction in cancer cells. $^{30}$ Some proteasome inhibitors, including bortezomib, bind and inhibit other $\beta$-subunits in conjunction with $\beta 5$, while other compounds bind the a-subunits or even to the enzymes involved in ubiquitination.

As of September 2011, the National Institutes of Health described 188 different clinical trials in a state of active recruitment, involving applications of the proteasome inhibitor bortezomib (Velcade) to cancer therapy and can be found at: http://clinicaltrials.gov/ct2/results?term $=$ bortezomib+cancer\&recr=Open\&no_unk=Y. However, common toxic side effects as well as native or acquired resistance to bortezomib has been described in human cancers and in various cell lines. For this reason, new classes of compounds are in continuous states of empirical design and analysis. Thirteen clinical trials are underway that include proteasome inhibitory compounds other than bortezomib and are described at: http://clinicaltrials.gov/ct $2 /$ results?term=proteasome + in hibitor+cancer+NOT+bortezomib\&recr=Open. The vast majority of these trials (combination therapies that include bortezomib or a variety of other proteasome inhibitors) are in the earliest state of human testing (phase I trials).

\section{Bortezomib}

Bortezomib is an FDA-approved reversible proteasome inhibitor that primarily functions as an inhibitor of b5 activity. ${ }^{40}$ The most common toxicities associated with bortezomib treatment include thrombocytopenia and neuropathy. ${ }^{2}$ Recent application of bortezomib in cancer therapy has focused on its efficacy as a combination agent for myeloma in which patients experienced improved time to progression and overall survival when treated with melphalan, prednisone, and bortezomib than when treated with melphalan and prednisone alone. ${ }^{41}$ Other studies highlight the addition of bortezomib to established therapies in newly diagnosed myeloma and include such combinations as lenalidomide and dexamethasone, ${ }^{41}$ melphalan, prednisone, and thalidomide, and dexamethasone, cyclophosphamide, and lenalidomide. ${ }^{42,43}$

While these studies describe the promise of bortezomib for myeloma and there have been successes in the treatment of mantle cell lymphoma, ${ }^{44}$ other malignancies, hematological and solid-tumor, have shown meager responses to bortezomib treatment. Furthermore, bortezomib response followed by resistance (refractory malignancy) remains a clinical problem. To identify mechanisms of resistance, the proteasomal $\beta 5$-subunit has been characterized in various cell culture models and was found to be mutated and/or over-expressed, ${ }^{45,46}$ indicating the potential for clinically relevant resistance mechanisms to bortezomib.

\section{Carfilzomib}

A variety of novel proteasome inhibitors have been developed to circumvent bortezomib resistance and potentially decrease toxicity. Carfilzomib is an irreversible inhibitor of the proteasomal $\beta 5$-subunit and is therefore more potent than the reversible bortezomib. ${ }^{47}$ Because of its irreversible nature, a cell must synthesize new proteins to form new proteasome molecules to overcome carfilzomib's mechanism of action. Importantly, carfilzomib is an effective proteasome inhibitor in certain cell lines resistant to bortezomib ${ }^{48,49}$ and in myeloma patients who display disease progression after bortezomib treatment. ${ }^{50}$ Carfilzomib is currently in phase II clinical trials and may become an important alternative to bortezomib 


\section{FOCUS: ADVANCES IN CLINICAL CANCER RESEARCH}

treatment in a variety of malignancies.

\section{Marizomib (NPI-0052)}

A second irreversible proteasome inhibitor, marizomib also inhibits the proteasomal b5-subunit in addition to the $\beta 1$ - and $\beta 2$-subunits. In cell culture and in mouse models of leukemia and myeloma, marizomib induces apoptosis and slows tumor cell proliferation. ${ }^{51,52}$ Prolonged treatment with marizomib, or with analogs with similar structure, has been suggested to promote apoptosis induction more potently in leukemia cells than compounds such as bortezomib because multiple proteasome activities are inhibited. ${ }^{53}$ Because of promising findings in early studies, marizomib is under continued investigation in phase I clinical trials for various malignancies that include, relapsed and/or refractory myeloma, non-small cell lung carcinoma, pancreatic cancer, and others.

\section{Nutritional Compounds}

The cancer-preventive and anticancer effects of dietary polyphenols and flavonoids such as those in green tea are supported by results from epidemiological, cell culture, and animal studies. Several of these plant polyphenols act as proteasome inhibitors and may sensitize tumor cells to traditional chemo- and radiation therapies by interfering in pathways that lead to drug resistance. ${ }^{54}$ The green tea polyphenols epigallocatechin3-gallate (EGCG) has proven an effective proteasome inhibitor in numerous cell-based and animal-model studies. ${ }^{55}$ Likewise, the soy isoflavone, Genistein, ${ }^{56}$ the active ingredient of tumeric, curcumin, ${ }^{57}$ the Chinese medicinal compound, Celastrol ${ }^{58}$ and several other flavonoids ${ }^{59}$ and nutritional compounds have all been implicated as specific and potent proteasome inhibitors. Compounds such as genistein, and green tea polyphenols have shown potential in phase II clinical trials. ${ }^{1}$

While nutritional compounds appear to possess promising anticancer effects, oral dietary polyphenols often exhibit poor bioavailability, possibly due to their inability to pass through the gut and into the circulation intact. Generally, the proteasome inhibitory activities of dietary polyphenols depend on their susceptibility to biotransformation reactions such as methylation, sulfonation, and glucuronidation. ${ }^{1}$ Additionally, it should be noted that while EGCG is an effective proteasome inhibitor on its own, EGCG at very high doses is antagonistic to compounds such as bortezomib in myeloma cell lines. ${ }^{60}$ While it is unlikely to be able to achieve a clinically relevant, bortezomib-inhibiting, dose of EGCG, it is an important issue that a full patient history is obtained when monitoring any therapy.

\section{CONCLUSIONS}

The Ub-proteasome pathway is responsible for the vast majority of proteolysis in eukaryotic cells and disruption of this pathway alters the normal concentration of cellcycle regulatory proteins, proteins involved in apoptosis, and ultimately, cellular homeostasis. The reversible proteasome inhibitor bortezomib has been successful as a treatment option for multiple myeloma and mantle cell lymphoma, but cancer recurrence is a major clinical problem. Interestingly, a novel strategy to overcoming bortezomib resistance focuses on inhibition of the enzymes that generate the ubiquitin tag. By blocking the activity of the E1, E2 and E3 enzymes, proteins that would normally be destined for proteasomal destruction accumulate and disturb cellular homeostasis. ${ }^{61}$ These novel concepts may lead to the development of new synthetic Ub-proteasome pathway inhibitors to achieve the best possible outcomes for cancer patients.

\section{REFERENCES}

1. Landis-Piwowar KR; Milacic V; Chen D, et al. The proteasome as a potential target for novel anticancer drugs and chemosensitizers. Drug resistance updates : reviews and commentaries in antimicrobial and anticancer chemotherapy 2006;9:263-73.

2. Richardson PG; Barlogie B; Berenson J, et al. A phase 2 study of bortezomib in relapsed, refractory myeloma. N Engl J Med 2003;348:2609-17.

3. Richardson PG; Sonneveld P; Schuster MW, et al. Bortezomib or high-dose dexamethasone for relapsed multiple myeloma. $\mathrm{N}$ Engl J Med 2005;352:2487-98.

4. Landis-Piwowar K; Chen D; Chan $\mathrm{TH}$, et al. Inhibition of catechol-Omicron-methyltransferase activity in human breast cancer cells enhances the biological effect of the green tea polyphenol (-)-EGCG. Oncol Rep 2010;24:563-9.

5. Glickman MH; Ciechanover A. The ubiquitin-proteasome proteolytic pathway: destruction for the sake of construction. Physiol Rev 2002;82:373-428.

6. Ciechanover A; Orian A; Schwartz AL. The ubiquitinmediated proteolytic pathway: mode of action and clinical implications. J Cell Biochem Supplement 2000;34:40-51.

7. Tanahashi N; Tsurumi C; Tamura T, et al. Molecular structure of 20S and 26S proteasomes. Enzyme Protein 1993;47:241-51.

8. Saeki Y; Kudo T; Sone T, et al. Lysine 63-linked polyubiquitin chain may serve as a targeting signal for the $26 \mathrm{~S}$ proteasome. Embo J 2009;28:359-71.

9. Groll M; Heinemeyer W; Jager S, et al. The catalytic sites of $20 S$ proteasomes and their role in subunit maturation: a mutational and crystallographic study. Proc Natl Acad Sci U S 


\section{FOCUS: ADVANCES IN CLINICAL CANCER RESEARCH}

A 1999;96:10976-83.

10. Voges D; Zwickl P; Baumeister W. The $26 \mathrm{~S}$ proteasome: a molecular machine designed for controlled proteolysis. Annu Rev Biochem 1999;68:1015-68.

11. Glotzer M; Murray AW; Kirschner MW. Cyclin is degraded by the ubiquitin pathway. Nature 1991;349:132-8.

12. Won KA; Reed SI. Activation of cyclin E/CDK2 is coupled to site-specific autophosphorylation and ubiquitin-dependent degradation of cyclin E. Embo J 1996;15:4182-93.

13. Diehl JA; Zindy F; Sherr CJ. Inhibition of cyclin D1 phosphorylation on threonine-286 prevents its rapid degradation via the ubiquitin-proteasome pathway. Genes Dev 1997;11:957-72.

14. Chen W; Lee J; Cho SY, et al. Proteasome-mediated destruction of the cyclin a/cyclin-dependent kinase 2 complex suppresses tumor cell growth in vitro and in vivo. Cancer Res 2004;64:3949-57.

15. Li B; Dou QP. Bax degradation by the ubiquitin/proteasomedependent pathway: involvement in tumor survival and progression. Proc Natl Acad Sci U S A 2000;97:3850-5.

16. Shringarpure R; Grune $\mathrm{T}$; Mehlhase $\mathrm{J}$, et al. Ubiquitin conjugation is not required for the degradation of oxidized proteins by proteasome. J Biol Chem 2003;278:311-8.

17. Ciechanover A. The ubiquitin-proteasome pathway: on protein death and cell life. The EMBO journal 1998;17:7151-60.

18. Ruschak AM; Slassi M; Kay LE, et al. Novel proteasome inhibitors to overcome bortezomib resistance. J Natl Med Assoc 2011;103:1007-17.

19. Sun J; Nam S; Lee CS, et al. CEP1612, a dipeptidyl proteasome inhibitor, induces p21WAF1 and p27KIP1 expression and apoptosis and inhibits the growth of the human lung adenocarcinoma A-549 in nude mice. Cancer Res 2001;61:1280-4.

20. Blagosklonny MV. P53: an ubiquitous target of anticancer drugs. Int J Cancer 2002;98:161-6.

21. Perkins ND. The Rel/NF-kappa B family: friend and foe. Trends in Biochem Sci 2000;25:434-40.

22. Green DR; Reed JC. Mitochondria and apoptosis. Science 1998;281:1309-12.

23. Miyashita T; Kitada S; Krajewski S, et al. Overexpression of the $\mathrm{Bcl}-2$ protein increases the half-life of p21Bax. J Biol Chem 1995;270:26049-52.

24. McConkey DJ; Chandra J; Wright S, et al. Apoptosis sensitivity in chronic lymphocytic leukemia is determined by endogenous endonuclease content and relative expression of BCL-2 and BAX. J Immunol 1996;156:2624-30.

25. Chang YC; Lee YS; Tejima $T$, et al. $\mathrm{mdm} 2$ and bax, downstream mediators of the $\mathrm{p} 53$ response, are degraded by the ubiquitin-proteasome pathway. Cell Growth Differ 1998;9:79-84.

26. Fecker LF; Geilen CC; Tchernev G, et al. Loss of proapoptotic $\mathrm{Bcl}-2$-related multidomain proteins in primary melanomas is associated with poor prognosis. J Invest Dermatol 2006;126:1366-71.

27. Liu FT; Agrawal SG; Gribben JG, et al. Bortezomib blocks Bax degradation in malignant $\mathrm{B}$ cells during treatment with TRAIL. Blood 2008;111:2797-805.

28. Drexler HC. Activation of the cell death program by inhibition of proteasome function. Proc Natl Acad Sci U S A 1997;94:855-60.

29. Drexler HC; Risau W; Konerding MA. Inhibition of proteasome function induces programmed cell death in proliferating endothelial cells. The FASEB journal : official publication of the Federation of American Societies for Experimental Biology 2000;14:65-77.

30. An B; Goldfarb RH; Siman R, et al. Novel dipeptidyl proteasome inhibitors overcome $\mathrm{Bcl}-2$ protective function and selectively accumulate the cyclin-dependent kinase inhibitor p27 and induce apoptosis in transformed, but not normal, human fibroblasts. Cell Death Differ 1998;5:1062-75.

31. Orlowski RZ; Eswara JR; Lafond-Walker A, et al. Tumor growth inhibition induced in a murine model of human Burkitt's lymphoma by a proteasome inhibitor. Cancer Res 1998;58:4342-8.

32. Soligo D; Servida F; Delia D, et al. The apoptogenic response of human myeloid leukaemia cell lines and of normal and malignant haematopoietic progenitor cells to the proteasome inhibitor PSI. Br J of Haematol 2001;113:126-35.

33. Landis-Piwowar KR; Huo C; Chen D, et al. A novel prodrug of the green tea polyphenol (-)-epigallocatechin-3-gallate as a potential anticancer agent. Cancer Res 2007;67:4303-10.

34. Rodriguez MS; Desterro JM; Lain S, et al. Multiple C-terminal lysine residues target $\mathrm{p} 53$ for ubiquitin-proteasome-mediated degradation. Mol Cell Biol 2000;20:8458-67.

35. Zhu Q; Wani G; Yao J, et al. The ubiquitin-proteasome system regulates p53-mediated transcription at p21waf1 promoter. Oncogene 2007;26:4199-208.

36. Lopes UG; Erhardt P; Yao R, et al. p53-dependent induction of apoptosis by proteasome inhibitors. J Biol Chem 1997;272:12893-6.

37. Khan MS; Halagowder D; Devaraj SN. Methylated chrysin induces co-ordinated attenuation of the canonical Wnt and NF-kB signaling pathway and upregulates apoptotic gene expression in the early hepatocarcinogenesis rat model. Chem Biol Interact 2011;193:12-21.

38. Landis-Piwowar KR; Milacic V; Dou QP. Relationship between the methylation status of dietary flavonoids and their growth-inhibitory and apoptosis-inducing activities in human cancer cells. J Cell Biochem 2008;105:514-23.

39. Osanai K; Landis-Piwowar KR; Dou QP, et al. A para-amino substituent on the D-ring of green tea polyphenol epigallocatechin-3-gallate as a novel proteasome inhibitor and cancer cell apoptosis inducer. Bioorg Med Chem 2007; 15:5076-82.

40. Crawford LJ; Walker B; Ovaa H, et al. Comparative selectivity and specificity of the proteasome inhibitors BzLLLCOCHO, PS-341, and MG-132. Cancer Res 2006;66:6379-86.

41. San Miguel JF; Schlag R; Khuageva NK, et al. Bortezomib plus melphalan and prednisone for initial treatment of multiple myeloma. N Engl J Med 2008;359:906-17.

42. Palumbo A; Bringhen S; Rossi D, et al. Bortezomib-melphalanprednisone-thalidomide followed by maintenance with bortezomib-thalidomide compared with bortezomibmelphalan-prednisone for initial treatment of multiple myeloma: a randomized controlled trial. J Clin Oncol 2010;28:5101-9.

43. Kumar SK; Flinn I; Noga SJ, et al. Bortezomib, dexamethasone, cyclophosphamide and lenalidomide combination for newly diagnosed multiple myeloma: phase 1 results from the multicenter EVOLUTION study. Leukemia 2010;24:1350-6.

44. Fisher RI; Bernstein SH; Kahl BS, et al. Multicenter phase II 


\section{FOCUS: ADVANCES IN CLINICAL CANCER RESEARCH}

study of bortezomib in patients with relapsed or refractory mantle cell lymphoma. J Clin Oncol 2006;24:4867-74.

45. Oerlemans R; Franke NE; Assaraf YG, et al. Molecular basis of bortezomib resistance: proteasome subunit beta5 (PSMB5) gene mutation and overexpression of PSMB5 protein. Blood 2008;112:2489-99.

46. Lu S; Yang J; Chen Z, et al. Different mutants of PSMB5 confer varying bortezomib resistance in $\mathrm{T}$ lymphoblastic lymphoma/leukemia cells derived from the Jurkat cell line. Exp Hematol 2009;37:831-7.

47. Parlati F; Lee SJ; Aujay M, et al. Carfilzomib can induce tumor cell death through selective inhibition of the chymotrypsin-like activity of the proteasome. Blood 2009;114:3439-47.

48. Demo SD; Kirk CJ; Aujay MA, et al. Antitumor activity of PR-171, a novel irreversible inhibitor of the proteasome. Cancer Res 2007;67:6383-91.

49. Kuhn DJ; Chen Q; Voorhees PM, et al. Potent activity of carfilzomib, a novel, irreversible inhibitor of the ubiquitinproteasome pathway, against preclinical models of multiple myeloma. Blood 2007;110:3281-90.

50. Jain S; Diefenbach C; Zain J, et al. Emerging role of carfilzomib in treatment of relapsed and refractory lymphoid neoplasms and multiple myeloma. Core Evid 2011;6:43-57.

51. Singh AV; Palladino MA; Lloyd GK, et al. Pharmacodynamic and efficacy studies of the novel proteasome inhibitor NPI0052 (marizomib) in a human plasmacytoma xenograft murine model. Br J Haematol 2010;149:550-9.

52. Chauhan D; Catley L; Li G, et al. A novel orally active proteasome inhibitor induces apoptosis in multiple myeloma cells with mechanisms distinct from Bortezomib. Cancer Cell
2005;8:407-19.

53. Miller CP; Manton CA; Hale R, et al. Specific and prolonged proteasome inhibition dictates apoptosis induction by marizomib and its analogs. Chem Biol Interact 2011.

54. Garg AK; Buchholz TA; Aggarwal BB. Chemosensitization and radiosensitization of tumors by plant polyphenols. Antioxid Redox Signal 2005;7:1630-47.

55. Dou QP; Landis-Piwowar KR; Chen D, et al. Green tea polyphenols as a natural tumour cell proteasome inhibitor. Inflammopharmacology 2008;16:208-12.

56. Kazi A; Daniel KG; Smith DM, et al. Inhibition of the proteasome activity, a novel mechanism associated with the tumor cell apoptosis-inducing ability of genistein. Biochem Pharmacol 2003;66:965-76.

57. Milacic V; Banerjee S; Landis-Piwowar KR, et al. Curcumin inhibits the proteasome activity in human colon cancer cells in vitro and in vivo. Cancer Res 2008;68:7283-92.

58. Yang H; Chen D; Cui QC, et al. Celastrol, a triterpene extracted from the Chinese "Thunder of God Vine," is a potent proteasome inhibitor and suppresses human prostate cancer growth in nude mice. Cancer Res 2006;66:4758-65.

59. Chen D; Daniel KG; Chen MS, et al. Dietary flavonoids as proteasome inhibitors and apoptosis inducers in human leukemia cells. Biochem Pharmacol 2005;69:1421-32.

60. Golden EB; Lam PY; Kardosh A, et al. Green tea polyphenols block the anticancer effects of bortezomib and other boronic acid-based proteasome inhibitors. Blood 2009;113:5927-37.

61. Xu GW; Ali M; Wood TE, et al. The ubiquitin-activating enzyme E1 as a therapeutic target for the treatment of leukemia and multiple myeloma. Blood 2010;115:2251-9. 